\title{
ARTIFICIAL INTELLIGENCE IN PUBLIC HEALTH: CHALLENGES IN DEVELOPING COUNTRIES
}

\author{
Madhur Mrinal \\ MPH, Laboratory and Molecular Epidemiology, \\ Amity Institute of Public Health
}

\begin{abstract}
Paper reviews the challenges of implementing Artificial Intelligence in developing countries. Further it focuses on some of the solutions to overcome these challenges and create awareness among the people for the applications of Artificial Intelligence in Public Health System.
\end{abstract}

\section{INTRODUCTION}

The term "Artificial Intelligence (AI)" was first introduced by John McCarthy in 1956. It was based on the concept of thinking machines but as this field progress, the definition of artificial intelligence keeps on changing. According to the modern dictionary artificial intelligence is defined as the sub-field of computer science that imitate human beings in various aspects. English Oxford Living dictionary termed Artificial Intelligence as the theory and development of computer systems able to perform tasks normally requiring human intelligence, such as visual perception, speech recognition, decisionmaking, and translation between languages. MeriamWebster, a famous scientist also defines artificial intelligence as the branch of computer science dealing with the simulation of intelligent behavior in computers and the capability of a machine to imitate intelligent human behavior (Marr,2018).

Several Technological industries such as I.B.M, Google, Apple and Microsoft comes together to work on artificial intelligence to benefit the society. It becomes very important part in present situation and it is highly use in every field we can imagen such as Marketing, Banking, Finance, Gaming, Health Care, Automobiles and Chatbots.

Artificial Intelligence in Public Health have already shown great results by the use of various applications such as integrated health information systems, patient education, geocoding health data, social media analytics, epidemic and syndromic surveillance, predictive modeling and decision support, mobile health, and medical imaging (Nejad et al, 2018). It also increases speed, accuracy and productivity in delivering Health Care Services to the society' eventually leads to the reduction of disease burden from the targeted population (Panda et al, 2018). In 2017, the United Nations (UN) convened a global meeting to discuss the development and deployment of AI applications to reduce poverty and deliver a broad range of critical public services (Geneva, 2017). Recently, another UN meeting brought together various stakeholders to assess the role AI could play in achieving the Sustainable Development Goals (see U.N).

\section{APPLICATIONS OF AI IN PUBLIC HEALTH:}

\section{Managing Electronic Medical Records (EMRs):}

Electronic Medical Records are the digital medical records of the patients based on the traditional paper medical records (Wahl, 2018) (Rouse,2011). These medical records can be collected at pharmacy, hospitals and even private clinics. AI provides the edge to the health workers to maintain EMRs of the individuals which can be access from anywhere around the globe using cloud computing. EMRs are also very important to the health professionals in collection of data for research in medical field and health informatics. Further, it can be used for surveillance of the chronic diseases (Chiolero et al ,2013).

\section{Digital Consultation:}

Digital consultations made its place in present situation because of the smartphones, apps like Babylon in UK use AI to give medical consultation to the users by analyzing the symptoms and history of the medical reports of the person comparing from the data base of illnesses. If the situation is worse than app advice to consult the physician personally, if not than it automatically prescribes effective treatment according to the symptoms $^{10}$. Another digital 


\section{International Journal of Engineering Applied Sciences and Technology, 2020 Vol. 5, Issue 4, ISSN No. 2455-2143, Pages 151-154 \\ Published Online August 2020 in IJEAST (http://www.ijeast.com)}

consultation that functions on $\mathrm{AI}$ is Buoy, symptom checker chatbot which uses canned response and help patient to choose from the options based on their health issue (Reynoso,2019).

\section{Doing repetitive jobs:}

Work like analyzing tests, CT scans, X-Rays and maintaining electronic medical records of the patients can be more accurate and much faster using AI. Radiology and Cardiology are the two disciplines which require huge amount of time but in future $\mathrm{AI}$ can also reduce this time. Cardiologists and Radiologist will look into the complex cases where human supervision will require (Navito,2015).

\section{Health Monitoring:}

Health monitoring mainly done through the use of wearable health trackers such as fitbit, apple watch, garmin, etc. The concept of Wearable Health Devices (WHDs) was introduced in 1990 to make the individual monitoring their own health and fitness which also referred as "Patient empowerment". These wearable keeps track of the health and fitness status of the user which is also important for early diagnosis of cardio vascular diseases and providing data to the clinicians for the treatment. Further, self-monitoring of health status leads to increase awareness of maintaining good health among the people (Dias and Cunha, 2018).

\section{Drug Development:}

Developing drugs through traditional methods of clinical trials requires more time and money. Hence, pharmaceutical industries are now accepting AI based system for creation of new drugs and acknowledge its abilities to address the therapeutic needs of the patients who suffer from viral diseases to complex diseases such as Cancer, Tuberculosis and Alzheimer's. AI based platform of drug development provide the better results to the pharmaceutical industries by cutting down the time and cost of the drugs (Reiss Robert, 2020). AI based drug development program was used in the time of the outbreak of Ebola virus to scan the existing medicines which can be redesign to reduce the effect of the virus and as a result AI program found two medicines which was very effective in controlling the outbreak and save many lives (Navito,2015).

\section{Challenges in implementing AI in Health Sector in Developing Countries:}

AI have huge potential and it keeps on increasing to the different branches of health care system. But there are few challenges that must be resolve before implementing AI base platform in health sector specially in developing countries.

Developing Countries are very new to the applications of AI as compared to the European and Western countries because of the smaller figure of professionals and AI developers (Mahajan et al, 2019). Most of the countries which are financially struggling are using AI based platform made by other countries which results in more expenditure of money in health sector and it leads to the financial burden for the country to spend on its health sector. Hence, these countries are limited to the use of AI in delivering health services.

Lack of awareness for adaptation and implementation of AI in health sector seems challenging for the medical professionals as they are using traditional methods for delivering health care services since the long time. Data privacy and security is also very important part of implementing AI platforms in health sector. Some AI platforms are responsible for sharing an individual medical records on global cloud computing system which make the patients personal information accessible globally even without the knowledge of the patient (McGrail Samantha,2020).

Another very big challenge of implementing AI in health care in developing countries specially in AsiaPacific region is due to the lack of availability and accessibility of internet services among the people and it is believed that the number of internet users are far less than compare to the European and Western countries. Mobile Health apps and online consultation are of no use for the people who lives in remote areas as they don't have the internet access and modern gadgets. Even the people living in mainstream areas are not aware about the emerging AI technology in health care sector which directly reduce the usage of mobile health and online consultation in developing countries (Mahajan et al, 2019).

There are less than $50 \%$ of the health professional that responded that they have been provided AI training from their organization and 67\% supports the adaptation of $\mathrm{AI}^{14}$. It requires the skill group to implement and use artificial intelligence in health sector and these skills are not taught in many parts of the industries which leads incompatibility of health workers with AI platforms (McGrail Samantha,2020). 


\section{International Journal of Engineering Applied Sciences and Technology, 2020 Vol. 5, Issue 4, ISSN No. 2455-2143, Pages 151-154 \\ Published Online August 2020 in IJEAST (http://www.ijeast.com)}

\section{CONCLUSION}

Artificial Intelligence, no doubt helps the human beings in every way which increases the rate of production, accuracy and reduce the time taken to complete the tasks in any field. AI technologies are highly use in Public Health Sectors specially in Wester and European countries while on the other hand, if we talk about developing countries such as India, Pakistan, Bangladesh, the use of AI found to be limited as per the population of the country.

There are number of challenges which are standing in the way of implementing AI in developing countries which were discussed above. The governments are now working with tech- companies for implementing the AI in health care system but they also need to focus on building its own AI technology for which they need to create awareness programs for the people and young generation and provide proper education system for designing and developing AI platforms. This will be a little investments for the proper institutions but it will give huge returns in a long run as the developing countries will have its own AI technology which can be applied in health system at less amount of cost and it makes the developing countries self-independent in terms of its AI based platforms. Further, it benefits the Government of developing countries by reducing the financial burden for health sector.

Sharing of personal data without the information of the patient is unethical, many companies which are providing AI technology are working towards the security of the personal data. Governments also introduce various guidelines for maintaining security of the personal or medical records of the patients, this will be the steps towards the cyber security of the data and it will make AI platforms more trustworthy.

Another challenge of developing countries is less accessibility and availability of internet, and without the use of internet the applications of AI are useless. Many telecommunication companies in the developing countries are working with the government to provide internet connectivity to people of the country, even in the remote areas at very low price which make the internet services affordable for maximum number of the population.

The future of Artificial Intelligence is bright, these are some of the challenges and in near future it will be resolve with the collaborative efforts of the AI companies and the governments. It will be really beneficial for the human beings when most of the population around the globe will able to use health services carried out by the artificial intelligence, it may also help to cure many diseases which have no cure at present. Further, it increases the research and production of new pharmaceutical medicines. Hence, awareness about AI in public health is very important and all the stakeholders should also focus on awareness programs for the people regarding Artificial Intelligence.

\section{REFERENCE}

1. AI for Good Global Summit, Geneva (2017). https://www.itu.int/en/ITU-T/AI/Pages/201706default.aspx . Accessed on 2020-07-04

2. Chiolero, Santschi and Paccaud,(2013), Public health surveillance with electronic medical records: at risk of surveillance bias and overdiagnosis, European Journal of Public Health, Volume 23, Issue 3, June 2013, Pages 350351, https://doi.org/10.1093/eurpub/ckt044 Accessed on 2020-07-11

3. Dias Duarte and Cunha(2018), Wearable Health Devices-Vital Sign Monitoring, Systems and Technologies, https://www.ncbi.nlm.nih.gov/pmc/articles/PMC6111 409/. Accessed on 2020-07-12

4. Mahajan, Vaidya, Gupta, Rane, Gupta(2019), Artificial intelligence in healthcare in developing nations: The beginning of a transformative journey, http://www.crstonline.com/article.asp?issn=25903233 ; year $=2019 ;$ volume $=2 ;$ issue $=2 ;$ spage $=182 ;$ epage $=189$; aulast=Mahajan\#top . Accessed on 2020-07-13

5. Marr, Bernard (2018), The Key Definitions of Artificial Intelligence (AI) That Explain Its Importance.

https://www.forbes.com/sites/bernardmarr/2018/02/1 4/the-key-definitions-of-artificial-intelligence-ai-thatexplain-its-importance/\#1ceeb904f5d8 . Accessed on 2020-07-02

6. McGrail Samantha (2020), Challenges of Artificial Intelligence Adoption in Healthcare, https://hitinfrastructure.com/news/challenges-ofartificial-intelligence-adoption-in-healthcare Accessed on 2020-07-14

7. Navito, 10 Common Applications of Artificial Intelligence in Health Care, https://novatiosolutions.com/10-commonapplications-artificial-intelligence-healthcare/ 


\section{International Journal of Engineering Applied Sciences and Technology, 2020 \\ Vol. 5, Issue 4, ISSN No. 2455-2143, Pages 151-154 \\ Published Online August 2020 in IJEAST (http://www.ijeast.com)}

\section{Accessed on 2020-07-11}

8. Panda, Prem Sagar and Bhatia Vikas, (Indian Journal of Family and community medicine vol. 4 issue 02, July-dec 2018), Role of Artificial Intelligence(AI) in Public Health, https://www.google.com/search?q=artificial+intellige nce+in+public+health\&rlz=1C1CHZL_enIN854IN85 $4 \&$ oq $=$ artificial $+\& a q s=$ chrome. $0.69 \mathrm{i} 5912 \mathrm{j} 0 \mathrm{j} 69 \mathrm{i} 57 \mathrm{j} 0 \mathrm{j} 6$ 9i6013.3401j0j7\&sourceid=chrome\&ie=UTF-8 . Accessed on 2020-07-03

9. Reynoso Rebecca (2019), AI in Healthcare $(+5$ Ways It's Used in 2020), https://learn.g2.com/ai-inhealthcare

10. Reiss Robert (2020), Transforming Drug Discovery Through Artificial Intelligence. https://www.forbes.com/sites/robertreiss/2020/03/03/ transforming-drug-discovery-through-artificialintelligence/\#7334fa656a15 . Accessed on 2020-0712

11. Rouse Margret, Electronic Medical Records(2011) https://whatis.techtarget.com/definition/electronicmedical-record-EMR . Accessed on 2020-07-10

12. Shaban-Nejad, A., Michalowski, M. \& Buckeridge, D.L. Health intelligence: how artificial intelligence transforms population and personalized health. $n p j \quad$ Digital Med 1,53 (2018). https://www.nature.com/articles/s41746-018-0058-9 . \begin{tabular}{lll}
\hline Accessed & on $2020-07-02$
\end{tabular}

13. U.N, Looking to future $\mathrm{UN}$ to consider how artificial intelligence could help achieve economic growth and reduce inequalities. https://www.un.org/sustainabledevelopment/blog/201 7/10/looking-to-future-un-to-consider-how-artificialintelligence-could-help-achieve-economic-growthand-reduce-inequalities/ Accessed on 2020-07-04

14. Wahl Brian, Aline Cossy-Gantner, Germann Stefan and R Schwalbe. (2018), Artificial intelligence (AI) and global health: how can AI contribute to health in resource-poor settings? BMJ Global Health. https://gh.bmj.com/content/3/4/e000798

Accessed on 2020-07-05 